\title{
Video Article \\ A Precise Pathogen Delivery and Recovery System for Murine Models of Secondary Bacterial Pneumonia
}

\author{
Timothy R. Borgogna ${ }^{1}$, Adrian Sanchez-Gonzalez ${ }^{2}$, Kelly Gorham ${ }^{2}$, Jovanka M. Voyich ${ }^{1}$ \\ ${ }^{1}$ Department of Microbiology and Immunology, Montana State University \\ ${ }^{2}$ University Communications, Montana State University
}

Correspondence to: Jovanka M. Voyich at jovanka@montana.edu

URL: https://www.jove.com/video/59566

DOI: doi:10.3791/59566

Keywords: Immunology and Infection, Issue 151, Intubation, intratracheal, lower respiratory tract, intranasal, pneumonia, influenza, Staphylococcus aureus, co-infection, superinfection

Date Published: 9/21/2019

Citation: Borgogna, T.R., Sanchez-Gonzalez, A., Gorham, K., Voyich, J.M. A Precise Pathogen Delivery and Recovery System for Murine Models of Secondary Bacterial Pneumonia. J. Vis. Exp. (151), e59566, doi:10.3791/59566 (2019).

\section{Abstract}

Secondary bacterial pneumonias following influenza infections consistently rank within the top ten leading causes of death in the United States. To date, murine models of co-infection have been the primary tool developed to explore the pathologies of both the primary and secondary infections. Despite the prevalence of this model, considerable discrepancies regarding instillation procedures, dose volumes, and efficacies are prevalent among studies. Furthermore, these efforts have been largely incomplete in addressing how the pathogen may be directly influencing disease progression post-infection. Herein we provide a precise method of pathogen delivery, recovery, and analysis to be used in murine models of secondary bacterial pneumonia. We demonstrate that intratracheal instillation enables an efficient and accurate delivery of controlled volumes directly and evenly into the lower respiratory tract. Lungs can be excised to recover and quantify the pathogen burden Following excision of the infected lungs, we describe a method to extract high quality pathogen RNA for subsequent transcriptional analysis. This procedure benefits from being a non-surgical method of delivery without the use of specialized laboratory equipment and provides a reproducible strategy to investigate pathogen contributions to secondary bacterial pneumonia.

\section{Video Link}

The video component of this article can be found at https://www.jove.com/video/59566/

\section{Introduction}

Secondary bacterial pneumonia following influenza infection is a leading cause of death in the United States and an active area of research ${ }^{1,2}$. Despite numerous studies using murine models of secondary bacterial pneumonia, inconsistencies regarding pathogen instillation and interrogation remain ${ }^{3,4,5}$. In addition, while many previous efforts have focused on the immunomodulatory effects of influenza that lead to an increased susceptibly to secondary bacterial infection, more recent data suggests virulence regulation of the bacterial pathogen is an equal contributor towards the establishment of disease ${ }^{6,7,8,9}$. These new data necessitate a more precise method to explore secondary bacterial pneumonia in murine models of co-infection that facilitate investigation into the pathogen response.

Unique to influenza co-infection models, host organisms are intentionally immunocompromised by a primary influenza infection prior to the administration of the secondary bacterial agent. In order to best replicate the disease pathogenesis observed in human hosts, it is imperative that the pathogen load of both primary and secondary agents be controlled so as to observe the individual and combinatorial effects of each infectious agent. Most commonly, respiratory infections in mice have been established through an intranasal administration ${ }^{3,4,5,6,10}$. Inasmuch as this route is noted for being technically simple and can be appropriate in some single-agent infection applications, it is unsuitable for co-infection models, as instillation procedures, dose volumes, and efficacy are highly variable within published literature . $^{3,4,5,6}$.

To gain a more complete understanding of secondary bacterial pneumonia pathogenesis, contributions of both the host and the pathogen must be considered. To that end, we have developed a straightforward and reproducible approach for recovery of viable bacteria and pathogen RNA from infected lungs. This method uses a simplified, non-invasive intratracheal instillation procedure followed by subsequent isolation of bacterial RNA. The intratracheal instillation procedure described herein is similar to previously described methods and is not limited to pathogen delivery ${ }^{11,12,13}$. Use of this particular procedure benefits from being low-cost and does not require the use of specialized equipment such as cannulas, guide wires, or fiber optic cable; furthermore, because this procedure is non-invasive it insures minimal stress on murine subjects, minimizes an inflammatory response from the inoculation mechanics, and provides an efficient delivery route for the infection of multiple subjects. Briefly, isoflurane anesthetized mice are suspended from the incisors. Forceps are used to gently grasp the tongue followed by insertion of a preloaded bent, blunt-tipped, 21-gauge needle into the trachea and delivery of pathogen load. Validation of this procedure is demonstrated by visual confirmation of dye equally distributed into the pulmonary compartment and recovery of bacterial load. We then demonstrate how to recover viable Staphylococcus aureus (S. aureus) from infected lungs and describe a reproducible method to isolate high quality pathogen RNA. 


\section{Protocol}

All methods conform to the National Institutes of Health guidelines and were approved by the Institutional Animal Care and Use Committee (IACUC) at Montana State University.

\section{Intratracheal Instillation}

1. Prepare a workspace containing the following supplies: an intubation platform, a sterile $1 \mathrm{~mL}$ syringe, sterile blunt-tipped forceps, sterile 21 gauge blunt-tipped needle, and two conical tubes to store the syringe and forceps.

NOTE: Intubation platforms can be purchased commercially or constructed in-house. The intubation platform used here was constructed using 0.25 inch plexiglass. Briefly, heat was applied to the plexiglass and the board is bent to an approximate $65^{\circ}$ interior angle. On the exterior side of the intubation platform, two machine screws were seeded 3 inches apart and a rubber-band was suspended between the two screws.

2. Prepare the pathogen inoculum by serially diluting the sample so that the desired final concentration is obtained in a total volume of $50 \mu \mathrm{L}$. Store the inoculum on ice.

3. Wearing sterile gloves, bend a $21 \mathrm{G}$ blunt-tipped needle to approximately $35^{\circ}$. NOTE: A separate needle is needed for each mouse.

4. Fix the bent $21 \mathrm{G}$ blunt-tipped needle to a $1 \mathrm{~mL}$ syringe. Draw a $100 \mu \mathrm{L}$ air cushion into the syringe followed by $50 \mu \mathrm{L}$ of the infectious agent. Place the loaded needle and syringe in a location easily accessible by the dominant hand.

5. Deeply anesthetize a mouse using a $4 \%$ isoflurane/oxygen mixture or similar IACUC approved method of anesthesia. Proper anesthesia depth is typically obtained when respiration rates slow to approximately 1 inhalation per $5-8 \mathrm{~s}$ and can be confirmed by pinching an appendage and observing no reaction from the mouse. NOTE: This method of anesthesia provided a sufficient anesthetization duration of approximately $1.5 \mathrm{~min}$. This was sufficient for members in our lab to learn and perform this instillation procedure; however, other institutionally approved anesthesia methods may be employed ${ }^{11,12,13}$.

6. Remove the anesthetized mouse from the anesthesia chamber and suspend the mouse on the intubation platform from the maxillary incisors.

7. To open a clear passage into the trachea, use the blunt-tipped forceps to gently grasp and extend the tongue outside of the mouth. Transfer the tongue from the forceps into the thumb and index finger of the non-dominant hand.

8. Remain holding the tongue in the non-dominant hand and pick up the pre-loaded syringe. Point the bent end of the needle away from the body and insert the needle into the oral cavity to the base of the tongue.

9. With the needle at the base of the tongue, gently angle the wrist to cause a slight push of the needle away from the body. This step insures the needle will be inserted into the trachea and not the esophagus.

10. Slowly guide the needle down into the trachea; often a slight tick is felt as the needle passes through the vocal fold. Pass the needle through the trachea until slight resistance is felt as the needle encounters the carina.

11. Slightly lift the needle in the proximal direction to suspend the needle above the carina. This enables delivery into the two primary bronchi. Fully depress the syringe's plunger to deliver the infectious load. Remove the needle from the trachea by lifting upward and discard.

12. Remove the mouse from the intubation platform by depressing the rubber band but maintain holding the mouse in an upright position. Obstruct the nasal airways by placing a finger directly over the nares. Hold this position for approximately 1 min or until several deep breathes are observed.

NOTE: This last step ensures that the total inoculum volume is delivered into the lower respiratory tract.

13. Return the mouse to its cage and observe a complete recovery from the anesthesia.

\section{Excision of Infected Lungs}

1. Working within a laminar flow hood to maintain sterility, prepare a workspace with the following items: a scale and sterile weigh boat, a dissection platform, a dissection kit containing scissors and forceps, sterile RNAse-free PBS, and sterile gauze.

2. Euthanize an infected mouse with $\mathrm{CO}_{2}$ or similar IACUC approved method of euthanasia.

3. Place an infected mouse on a dissection platform and secure the mouse using pins at the end of each appendage. Spray the mouse with ethanol to help maintain sterility of the working surfaces.

4. Beginning at the umbilicus, use a pair of forceps to lift the skin and make an incision up to the base of the trachea. Grasping the skin on either side of the initial incision, pull the skin away from the body and cut through the tissue connections. NOTE: It can be helpful to make several lateral cuts across the skin to reveal more of the thoracic region.

5. Starting at the base of the xiphoid process, make a small incision with the intention of puncturing the diaphragm. This results in an increase in pressure in the thoracic cavity that causes the lungs to retract. With the lungs retracted continue the incision to free the diaphragm.

6. Remove the ribs by making an incision along both sides of the rib cage. This will fully expose the thoracic cavity and lungs.

7. To excise the lungs, grasp the base of the heart with the forceps and lift upward. Place the scissors behind the lungs and begin to make small incisions through the tissue connections while continuing to the lift the heart upward.

8. Once the lungs have been excised, place them onto the sterile gauze and remove the heart. The heart can be easily removed by lifting it away from the lungs with the forceps and cutting the remaining tissue connections.

9. Transfer the lungs to a pre-tared weigh boat and record the weight.

10. After the lungs have been weighed, transfer them into sterile phosphate buffered saline (PBS) and temporarily store them on ice until moving forward to the pathogen recovery and analysis steps.

\section{Pathogen Recovery and Analysis}

1. Continuing to work within a laminar flow hood, prepare a workspace with the following supplies: a tissue grinder, sterile RNAse-free PBS, Buffer RLT supplemented with ß-mercaptoethanol (1:100), and 1.5 mL RNAse-free microcentrifuge tubes. 
CAUTION: ß-mercaptoethanol can be hazardous in the case of skin contact, ingestion, eye contact, or inhalation. Dilution of ßmercaptoethanol into buffer RLT should be done in a fume hood.

2. Begin by first adding $1 \mathrm{~mL}$ of sterile RNAse-free PBS to the tissue grinder. Once filled, store the tissue grinder on ice.

3. Prepare a series of sterile RNAse-free serial dilution tubes that will be used to quantify the bacterial load recovered from the infected lungs. This is most easily accomplished by aliquoting $900 \mu \mathrm{L}$ of sterile water into each microcentrifuge tube followed by serial dilution of the pathogen inoculum.

NOTE: The number of dilution tubes necessary for accurate pathogen enumeration will vary on initial pathogen input and duration of infection. This must be empirically determined.

4. Using sterile forceps, transfer the lungs into the prepared tissue grinder and thoroughly homogenize the tissue by pressing down on the pedestal while rotating.

5. Open the tissue grinder and aliquot $100 \mu \mathrm{L}$ of the homogenized sample into the first of the prepared serial dilution tubes. Serially dilute the samples and enumerate CFUs by plating onto nutrient agar. CFUs can be recorded as CFUs $/ \mathrm{mL}$ or CFUs $/ \mathrm{mL} / \mathrm{mg}$ of lung tissue. NOTE: At this step an additional $200 \mu \mathrm{L}$ of the homogenized sample can be removed for purification of viral RNA (see Table of Materials) or stored at $-80^{\circ} \mathrm{C}$ for future analysis.

6. After the aliquots have been removed for CFU determination and/or viral RNA isolation, replace the grinding pedestal and pellet the homogenized tissue by centrifugation at $4,000 \mathrm{rpm}(3724 \times \mathrm{g})$ for $10 \mathrm{~min}$ at $4{ }^{\circ} \mathrm{C}$.

7. Using a pipette, remove the supernatant from the pelleted sample and place into a $1.5 \mathrm{~mL}$ microcentrifuge tube.

NOTE: The supernatant is free of bacteria and can be stored at $-80^{\circ} \mathrm{C}$ for analysis of soluble host and secreted pathogen factors.

8. Resuspend the homogenized tissue pellet by pipetting or vortexing in $700 \mu \mathrm{L}$ of buffer RLT-ß-mercaptoethanol and transfer the sample to a sterile RNAse-free $1.5 \mathrm{~mL}$ microcentrifuge tube.

NOTE: At this point the samples can be stored at $-80^{\circ} \mathrm{C}$ for several months.

9. Purify RNA using slight modifications to the manufacturer's protocol of a purification kit (see Table of Materials); see Voyich et al. $2008^{14}$

1. Transfer the homogenized lung slurry into a $2 \mathrm{~mL}$ microcentrifuge tube containing $0.1 \mathrm{~mm}$ silica beads and processed via a bead beater for $20 \mathrm{~s}$ at $6 \mathrm{~m} / \mathrm{s}$.

2. Centrifuge the sample at $1,500 \mathrm{rpm}$ for 3 minutes. After centrifugation, pipette the supernatant into new $1.5 \mathrm{~mL}$ microcentrifuge tube.

3. Add $350 \mu \mathrm{L}$ of $96 \%-100 \%$ ethanol to the sample and thoroughly mix by pipetting or inversion.

4. Transfer $700 \mu \mathrm{L}$ of the sample to a purification column placed in a $2 \mathrm{~mL}$ collection tube. Centrifuge the sample at $\geq 8,000 \times g$ for $15 \mathrm{~s}$ and discard the flow-through. Run any excess sample through the same column as described above.

5. Wash the column with $700 \mu \mathrm{L}$ of buffer RW1 and centrifuge sample at $\geq 8,000 \times g$ for $15 \mathrm{~s}$. Discard the flow-through and transfer the silica membrane column into a new $2 \mathrm{~mL}$ collection tube.

6. Apply $500 \mu \mathrm{L}$ of buffer RPE to the column. Wash the column by centrifugation at $\geq 8,000 \times g$ for $15 \mathrm{~s}$. Discard the flow-through.

7. Apply an additional $500 \mu \mathrm{L}$ of buffer RPE to the RNeasy column and centrifuge at $8,000 \times g$ for 2 min. Discard flow-through and centrifuge the column at $\geq 10,000 \times g$ for $1 \mathrm{~min}$.

8. To elute purified RNA, begin by transferring the column to a new $1.5 \mathrm{~mL}$ RNAse-free microcentrifuge tube. Pipette $50 \mu \mathrm{L}$ of RNase-free water directly onto the silica-gel membrane of the column and centrifuge at $\geq 8000 \times g$ for $1 \mathrm{~min}$. NOTE: The eluted RNA can be run over the same column again to increase RNA yield.

9. Add $50 \mu \mathrm{L}$ of RNase-free water to the purified RNA to bring the total volume to $100 \mu \mathrm{L}$. Aliquot $350 \mu \mathrm{L}$ RLT-ß-mercaptoethanol followed by $250 \mu \mathrm{L}$ of $96 \%-100 \%$ ethanol to the sample and mix thoroughly by pipetting or inversion.

10. Apply the sample to a purification column placed in a collection tube and centrifuge at $\geq 8000 \times g$ for $15 \mathrm{~s}$. Discard the flow-through and replace the collection tube.

11. Wash the column by adding $350 \mu \mathrm{L}$ of buffer RW1 followed by centrifugation at $\geq 8000 \times g$ for $15 \mathrm{~s}$.

12. Prepare a DNase solution containing approximately 27 Kunitz units by adding $10 \mu \mathrm{L}$ of DNase stock ( $750 \mathrm{Kunitz}$ units $/ \mathrm{mL})$ to 70 $\mu \mathrm{L}$ of buffer RDD. Add $80 \mu \mathrm{L}$ of DNase solution directly onto the silica-gel membrane and incubate the sample for $15 \mathrm{~min}$ at room temperature.

13. Wash the column with $350 \mu \mathrm{L}$ of buffer RW1 and centrifuge at $\geq 8000 \times g$ for $15 \mathrm{~s}$ and discard the flow-through.

14. Repeat steps 3.9.6-3.9.8 to purify RNA.

NOTE: It is recommended to repeat the RNA clean-up procedure by following steps 3.9.9-3.9.10 and 3.9.6-3.9.8 (skip DNase steps 3.9.11-3.9.13). This additional clean-up it results in very high-quality RNA.

10. RNA yield can be quantified using a spectrophotometer with readings at $260 \mathrm{nM}$ for determining concentration and $260: 280 \mathrm{nM}$ for purity. After RNA yield is obtained, standardize the concentration of each RNA sample to $50 \mathrm{ng} / \mathrm{\mu L}$.

NOTE: It is recommended to make multiple aliquots at a concentration of $50 \mathrm{ng} / \mathrm{\mu L}$ to avoid freeze/thaw cycles that can lead to RNA degradation.

11. Store the purified RNA at $-80^{\circ} \mathrm{C}$ or use immediately for transcript analysis.

NOTE: In previous publications RNA from 3-5 mice was pooled for transcript analysis. Through optimization of the above technique, RNA from 1 mouse has been empirically determined as sufficient for transcript analysis $(80-120 \mathrm{ng} / \mu \mathrm{L})$.

\section{Representative Results}

Figure 1 utilizes a $0.1 \%$ weight/volume Coomassie brilliant blue solution to demonstrate that an intratracheal instillation delivers the inoculum directly and evenly within the lower respiratory tract. Figure 2 shows that the bacteria ( $S$. aureus) CFUs recovered directly from homogenized lung tissue. Figure 3 demonstrates the use of this system for precise delivery and recovery of inoculum in the lower respiratory tract by plotting the input and recovery CFUs from individual mice. Figure 4 shows the qRT-PCR amplification curve of the bacterial housekeeping gene gyrB to demonstrate that bacterial RNA can be extracted directly from infected lung tissue with minimal DNA contamination. Figure 5 shows the construction of a standard curve using qRT-PCR amplification of the influenza A virus M-segment to demonstrate viral RNA can be extracted directly from infected lung tissue. 


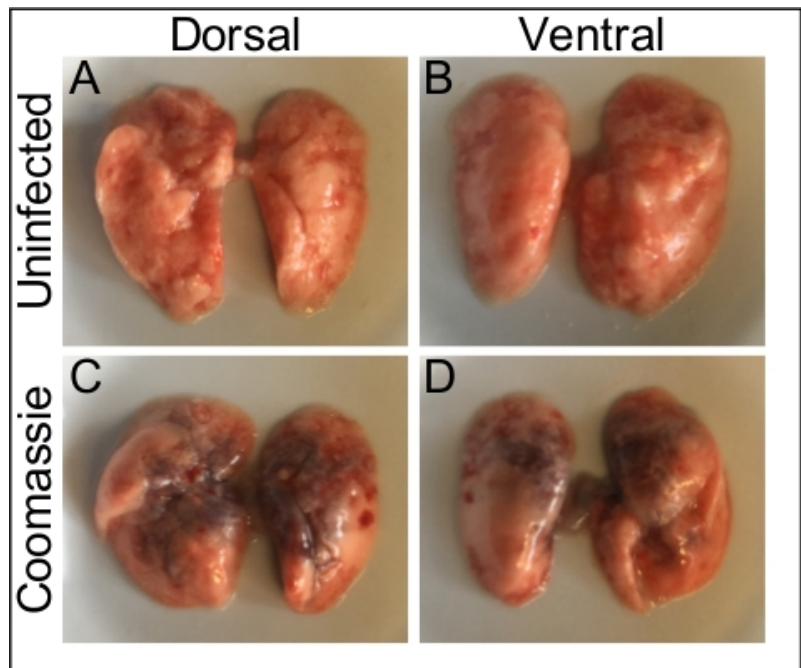

Figure 1: Intratracheal instillation enables even distribution into the lower respiratory tract. (A, B) Uninfected lungs were excised from a healthy mouse following intratracheal instillation of sterile PBS and photographed from (A) dorsal and (B) ventral perspectives. (C, D) $50 \mu \mathrm{L}$ of a $0.1 \%$ Coomassie brilliant blue solution were administered to an anesthetized mouse via intratracheal administration. (C) Dorsal. (D) Ventral. Please click here to view a larger version of this figure.

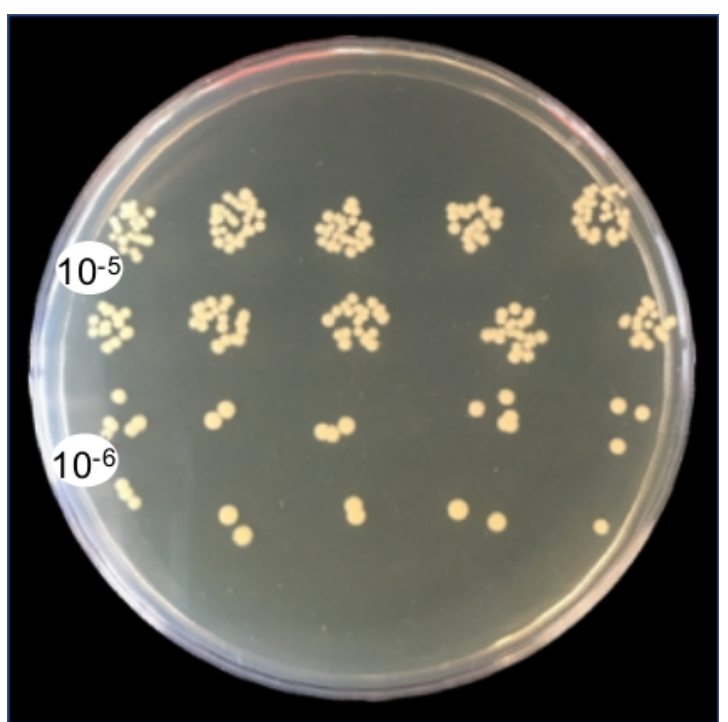

Figure 2: Representative recovery of bacterial CFUs from infected lung homogenate. Lungs were excised one day post-challenge with $S$. aureus. Following homogenation, $100 \mu \mathrm{L}$ of the lung slurry was serially diluted through $10^{-6}$. To enumerate CFUs recovered, $10 \mu \mathrm{L}$ drops were plated from the $10^{-5}$ and $10^{-6}$ dilutions onto tryptic soy agar (TSA) and incubated at $37^{\circ} \mathrm{C}$ with $5 \% \mathrm{CO}_{2}$ overnight. Please click here to view a larger version of this figure. 


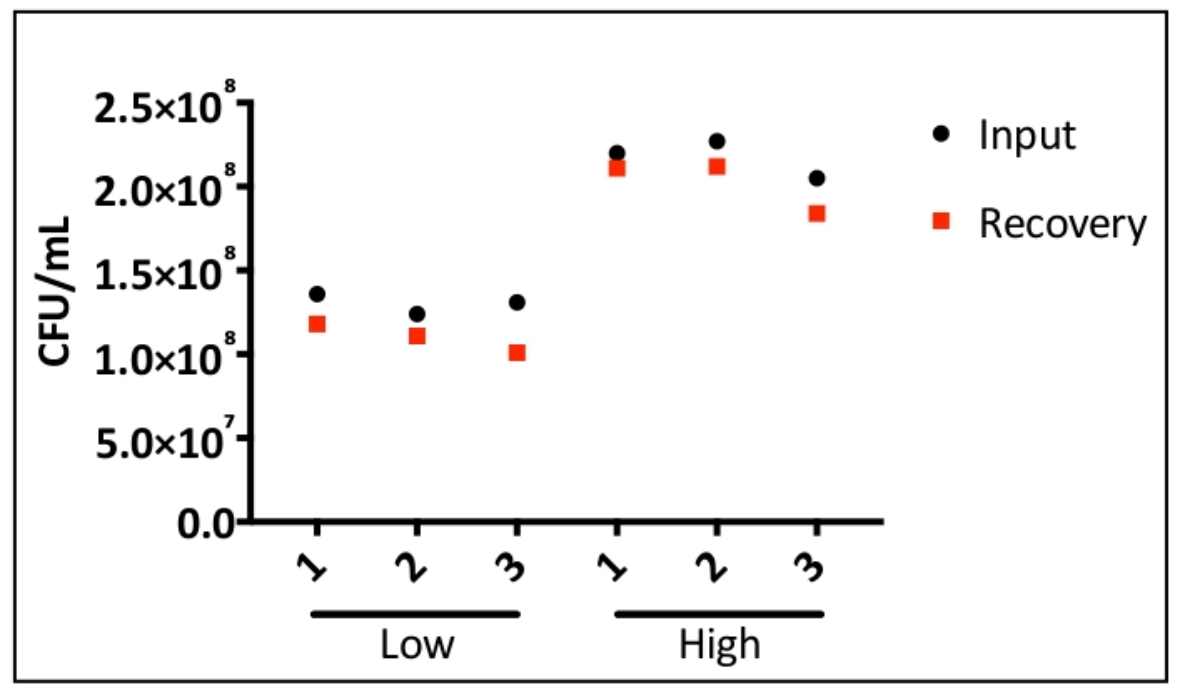

Figure 3: Precise delivery and recovery of the pathogen inoculum following intratracheal administration. Mice were divided into two groups containing three mice per group. Mice were subjected to intratracheal instillation of $S$. aureus at $1 \times 10^{8}$ (low) and $2 \times 10^{8}$ (high) $\mathrm{CFU} / \mathrm{mL}$. One hour post-infection, mice were euthanized and the lungs were excised to demonstrate the precision of the instillation and recovery. Bacterial inoculum and bacteria recovered from the lung homogenate were plated on TSA (tryptic soy agar). No significant differences were reported between bacterial input and recovery. Please click here to view a larger version of this figure.

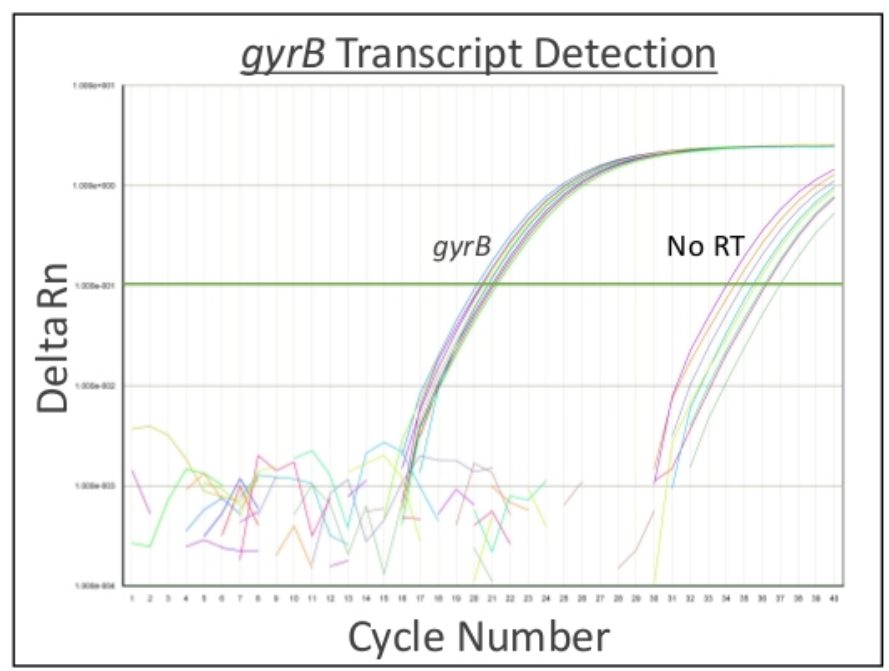

Figure 4: Representative bacterial RNA recovery and purity. Six-hours following intratracheal instillation with $1 \times 10^{8} \mathrm{CFU} / 50 \mu \mathrm{L}$ of $\mathrm{S}$. aureus, mice were euthanized. Lungs were excised and homogenized followed by resuspension of the lung slurry in buffer RLT-ß-mercaptoethanol. RNA was purified as described in step 3.9 ${ }^{14}$. qRT-PCR was used to detect transcripts of the bacterial housekeeping gene gyrB. A control containing no reverse transcriptase ( $\mathrm{nRT}$ ) was included to demonstrate the purity of the RNA recovered. At a threshold of 0.1, gyrB transcripts were detected at an average cycle of $21.1,20.3$, and 20.5. nRT controls were not detected until cycle averages $35.9,35.5$, and 35.0 . $n=3$ biological replicates, containing 3 technical replicates/biological replicate. Please click here to view a larger version of this figure. 


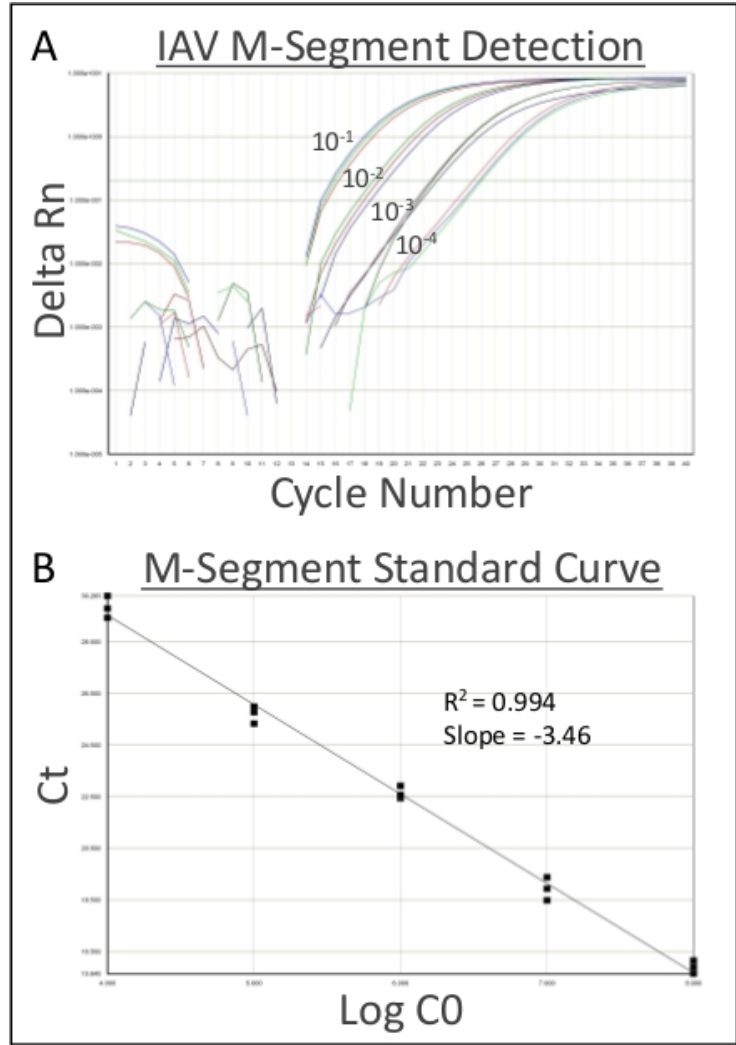

Figure 5: Representative viral RNA recovery. Six days following intratracheal instillation with $100 \mathrm{PFU} / 50 \mu \mathrm{L}$ of influenza A/PR/8/1934(H1N1), mice were euthanized. Lungs were excised and homogenized, and $200 \mu \mathrm{L}$ of the homogenized slurry was collected and passed through a 70 $\mu \mathrm{m}$ cell strainer prior to viral RNA purification. Purified RNA was serially diluted $\left(10^{-1}-10^{-4}\right)$ followed by amplification of influenza A M-segment. (A) Amplification plot of influenza A RNA recovered from an infected lung and diluted $10^{-1}-10^{-4}$. (B) Standard curve of influenza A M-segment. Threshold $=0.2, R^{2}=0.994$, slope $=-3.46$. Please click here to view a larger version of this figure

\section{Discussion}

Use of this model provides a highly efficient and reproducible method to study secondary bacterial infections. The ability to tightly control the delivery of the pathogen inoculum enables more precise observations of the individual and combinatorial effects of each pathogen. Inefficiencies in the more common intranasal instillation route have likely contributed to the discrepancies in dose volumes and concentrations present in the literature. It is reasonable that the lack of a precise murine system to study secondary bacterial pneumonia has delayed findings identifying bacterial specific responses that contribute to the severity of pulmonary co-infections. Developing a reproducible model to study virulence expression during secondary bacterial infections could lead to the identification of vaccine or drug targets to ameliorate these infections.

The intratracheal instillation step is critical to successfully establish a lower respiratory tract infection and any down-stream analysis of the pathogens. When learning this technique, it may be helpful to practice using a dye (as described in the methods) prior to administering infectious material. Using a dye allows for the direct visualization of the inoculum into the respiratory tract. A common mistake that can occur is insertion of the blunt-needle into the esophagus rather than the trachea. This will result in delivery of the inoculum into the stomach rather than the lungs. To correct this mistake, angle the needle further away from the body and pass it down into the trachea. Once mastered, this procedure is very efficient and can be used to conduct experiments with large numbers of mice. Working in batches to anesthetize mice, the intratracheal instillation can be completed in approximately 30 seconds per mouse. In addition, the excision of the lungs can be completed in 2 to 3 minutes per mouse.

Recovery of viable and pure bacterial RNA from infected tissues is critical for transcript analysis. RNases are ubiquitous and can quickly ruin an experiment ${ }^{15}$. Some methods include using RNase inhibitors; however, we have found that freezing the sample at $-80^{\circ} \mathrm{C}$ in $\mathrm{RLT}-\beta$ mercaptoethanol or immediately processing the sample for RNA isolation using all RNase free tubes and reagents are effective at reducing RNase contamination. Additionally, we recommend that a maximum of six samples be purified at one time. Including more than six samples can result in prolonged latencies between protocol steps that can culminate in RNA degradation. Once purified, care should also be taken to avoid any unnecessary freeze-thaw cycles. Thus, if multiple analyses will be done on one sample, aliquoting purified RNA for storage at $-80{ }^{\circ} \mathrm{C}$ is recommended.

In addition to the techniques reviewed herein, this method can be supplemented by performing bronchial alveolar lavage prior to the excision and homogenation of the lungs ${ }^{16}$. This can be accomplished by lavage of the entire lower respiratory tract or by using suture thread to restrict one branching arm of the bronchial tree followed by lavage through the remaining branch. Often this leads to a reduction in the recovery of the pathogen load but provides a sample whereupon information such as lactate dehydrogenase activity, cellular population identity, and cytokine 
profiles can be obtained ${ }^{16}$. Together these data can form a more complete understanding of the host-pathogen interactions occurring during secondary bacterial pneumonia.

While the methods discussed have been within the context of secondary bacterial pneumonia, they are suitable to be extended to any murine model of lower respiratory infection; specifically, those that would benefit from tightly controlled delivery and recovery of the installed inoculum. Furthermore, like many other infection routes, the intratracheal instillation can be utilized in non-infectious applications, such as the administration of therapeutics and environmental compounds ${ }^{12}$.

\section{Disclosures}

The authors have nothing to disclose.

\section{Acknowledgments}

The authors would to thank Nicole Meissner, M.D./Ph.D., Montana State University, for her help in establishing the intratracheal instillation method. This work was supported by the U.S. National Institutes of Health (Grants NIH-1R56AI135039-01A1, GM110732, R21AI128295, U54GM115371), as well as funds from the Montana University System Research Initiative (51040-MUSRI2015-03) and Montana State University Agriculture Experiment Station.

\section{References}

1. Xu, J., Murphy, S.L., Kochanek, K.D., Bastian, B.A. National Vital Statistics Reports Deaths: Final Data for 2013. National Center for Health Statistics. 64 (2), 1-119, at <http://www.cdc.gov/nchs/data/nvsr/nvsr64/nvsr64_02.pdf> (2016).

2. Morris, D.E., Cleary, D.W., Clarke, S.C. Secondary Bacterial Infections Associated with Influenza Pandemics. Frontiers in Microbiology. 8, 1041 (2017).

3. Lee, M., Arrecubieta, C., Martin, F.J., Prince, A., Borczuk, A.C., Lowy, F.D. A Postinfluenza Model of Staphylococcus aureus Pneumonia. The Journal of Infectious Diseases. 201 (4), 508-515 (2010).

4. Shepardson, K.M. et al. Differential Type I Interferon Signaling Is a Master Regulator of Susceptibility to Postinfluenza Bacterial Superinfection. mBio. (2016).

5. Miller, M.A. et al. Visualization of Murine Intranasal Dosing Efficiency Using Luminescent Francisella tularensis: Effect of Instillation Volume and Form of Anesthesia. PLOS ONE. 7 (2), e31359, at <https://doi.org/10.1371/journal.pone.0031359> (2012).

6. Robinson, K.M. et al. Influenza a virus exacerbates staphylococcus aureus pneumonia in mice by attenuating antimicrobial peptide production. Journal of Infectious Diseases. 209 (6), 865-875 (2014).

7. Reddinger, R.M., Luke-Marshall, N.R., Hakansson, A.P., Campagnari, A.A. Host physiologic changes induced by influenza a virus lead to Staphylococcus aureus biofilm dispersion and transition from asymptomatic colonization to invasive disease. $m B i o .7$ (4) (2016).

8. Borgogna, T.R. et al. Secondary Bacterial Pneumonia by Staphylococcus aureus Following Influenza A Infection Is SaeR/S Dependent. The Journal of Infectious Diseases. 218 (5), 809-813, at <http://dx.doi.org/10.1093/infdis/jiy210> (2018).

9. Shahangian, A. et al. Type I IFNs mediate development of postinfluenza bacterial pneumonia in mice. Journal of Clinical Investigation. 119 (7), 1910-1920 (2009).

10. McAuley, J.L. et al. Expression of the 1918 Influenza A Virus PB1-F2 Enhances the Pathogenesis of Viral and Secondary Bacterial Pneumonia. Cell Host and Microbe. 2 (4), 240-249 (2007).

11. Hamacher, J. et al. Microscopic wire guide-based orotracheal mouse intubation: Description, evaluation and comparison with transillumination. Laboratory Animals. 42 (2), 222-230 (2008).

12. Lawrenz, M.B., Fodah, R.A., Gutierrez, M.G., Warawa, J. Intubation-mediated Intratracheal (IMIT) Instillation: A Noninvasive, Lung-specific Delivery System. Journal of Visualized Experiments. (93), e52261 (2014).

13. Cai, Y., Kimura, S. Noninvasive Intratracheal Intubation to Study the Pathology and Physiology of Mouse Lung. Journal of Visualized Experiments. (81), e50601 (2013).

14. Voyich, J.M., Sturdevant, D.E., DeLeo, F.R. Analysis of Staphylococcus aureus gene expression during PMN phagocytosis. Methods in molecular biology (Clifton, N.J.). 431, 109-122 (2008).

15. Dyer, K., Rosenberg, H. The RNase a superfamily: Generation of diversity and innate host defense. Molecular Diversity. 10 (4), 585-597 (2006).

16. Van Hoecke, L., Job, E.R., Saelens, X., Roose, K. Bronchoalveolar Lavage of Murine Lungs to Analyze Inflammatory Cell Infiltration. Journal of Visualized Experiments. (123), e55398 (2017). 